\title{
THE MESH TRANSFORMATION METHOD AND OPTIMAL FINITE ELEMENT SOLUTIONS
}

\author{
ZHIPING LI \\ LMAM \& SCHOOL OF MATHEMATICAL SCIENCES, \\ PEKING UNIVERSITY, BEIJING 100871, P.R.CHINA
}

\begin{abstract}
Since the accuracy of finite element solutions of partial differential equations is generally mesh dependent, especially when solutions have singularities and discontinuities, a proper mesh generation is often important and sometimes crucial for an accurate numerical approximation of such problems. In this paper, the mesh transformation method is applied to the boundary value problems of elliptic partial differential equations, and it is proved that the method leads to the optimal finite element solutions.
\end{abstract}

\section{INTRODUCTION}

Consider the problem of minimizing an energy functional

$$
F(u ; \Omega)=\int_{\Omega} W(x, u(x), \nabla u(x)) d x
$$

in a set of admissible deformations

$$
\mathbb{U}\left(u_{0} ; \Omega\right)=\left\{u \in W^{1, p}\left(\Omega ; R^{m}\right): u=u_{0}, \text { on } \partial \Omega_{0}\right\},
$$

where $\Omega \subset R^{n}, n=2$ or 3 , is a bounded open set with a Lipschitz continuous boundary $\partial \Omega, \partial \Omega_{0}$ is a subset of $\partial \Omega$ with positive $(n-1)$-dimensional measure. It is well known that, when the energy density $W(x, u, v)$ satisfies certain general conditions on regularity, growth and coerciveness, and if $W(x, u, \cdot)$ is convex, then the problem is solvable [1] and the solutions can be approximated by the finite element method [2].

1991 Mathematics Subject Classification. 73C50,65K10,65N12,65N30.

Key words and phrases. energy minimization, mesh transformation, mesh quality control, optimal finite element solutions.

The research was supported in part by the Special Funds for Major State Basic Research Projects (2005CB321701), NSFC projects (10431050, 10571006 and 10528102) and RFDP of China. 
Since the accuracy of finite element solutions is generally mesh dependent, especially when solutions have singularities and discontinuities, a proper mesh generation is often important and sometimes crucial for an accurate numerical approximation of such problems. To reduce the mesh dependent of the finite element approximations, it is natural to involve the mesh distribution into the minimization procedure. The mesh transformation method was first introduced to compute microstructures in non-convex problems $[3,4,5]$. The idea of the mesh transformation method, which is to minimize the energy functional on all admissible finite element function spaces obtained by mesh distribution transformation, leads to the following discrete problem

$$
(M T M)\left\{\begin{array}{l}
\text { find }\left(\bar{u}_{h}, g_{h}\right) \in \mathbb{U}_{h}\left(u_{0} \circ g_{h} ; D\right) \times T_{h}(D) \text { such that } \\
F\left(\bar{u}_{h}, g_{h} ; D\right)=\inf _{(\bar{u}, g) \in \mathbb{U}_{h}\left(u_{0} \circ g ; D\right) \times T_{h}(D)} F(\bar{u}, g ; D),
\end{array}\right.
$$

where $D$ is the computation domain,

$$
\begin{gathered}
\mathbb{U}_{h}\left(\bar{u}_{0} ; D\right)=\left\{\bar{u} \in(C(\bar{D}))^{m}:\left.\bar{u}\right|_{\partial D_{0}}=\bar{u}_{0}, \text { and }\left.\bar{u}\right|_{K} \text { is affine } \forall K \in \mathfrak{T}_{h}(D)\right\} \\
T_{h}(D)=\left\{g \in T(D):\left.g\right|_{K} \text { is affine } \forall K \in \mathfrak{T}_{h}(D)\right\}
\end{gathered}
$$

with $\mathfrak{T}_{h}(D)$ being regular triangulations of $D$ with mesh size $h[2]$ and

$$
\begin{array}{r}
T(D)=\left\{\text { bijections } g: \bar{D} \rightarrow \bar{\Omega} \mid g \in W^{1, \infty}(D ; \Omega), g^{-1} \in W^{1, \infty}(\Omega ; D),\right. \\
\left.g\left(\partial D_{0}\right)=\partial \Omega_{0}, \text { and } \operatorname{det} \nabla g>0, \text { a.e. in } D\right\}
\end{array}
$$

and where the functional $F(\cdot, \cdot ; D)$ is defined by

$$
\left.F(\bar{u}, g ; D)=\int_{D} W\left(g(\bar{x}), \bar{u}(\bar{x}), \nabla \bar{u}(\bar{x})(\nabla g(\bar{x}))^{-1}\right)\right) \operatorname{det} \nabla g(\bar{x}) d \bar{x} .
$$

By setting

$$
x=g(\bar{x}), \quad u(x)=\bar{u}\left(g^{-1}(x)\right),
$$

it is easily seen that

$$
F(\bar{u}, g ; D)=F(u ; \Omega) .
$$

Compared with the standard finite element method which works on a finite element function space defined on a fixed finite element mesh, the mesh transformation method is actually trying to minimize the energy among finite element functions defined on all admissible finite element mesh distributions. In other words, the solution given by the mesh transformation method attains the lowest 
energy level among all admissible finite element function spaces defined by (1.5) and (1.6), and it is in such a sense that the solution is said to be the optimal finite element approximation. In fact, it is shown in section 2 that, for conforming finite element approximations of standard elliptic boundary value problems, the lowest energy is equivalent to the least error in the energy norm.

In section 3, the implementation of the mesh transformation method by the conjugate gradient method is discussed, where a mesh quality control term $F_{q}(\bar{u}, g ; D)$, which takes into consideration of conformity (or isotropy) and uniformity (or equi-distribution) $[6,7]$ of the mesh distribution as well as a penalty term on the relative element volume $\operatorname{det}(\nabla g)$ tending to either zero or infinity, is added to the energy functional $F(\bar{u}, g ; D)$ to regularize the mesh transformation procedure [8]. More sophisticated applications of the mesh transformation method, such as to work on an initial mesh created by the mesh adaption or moving mesh method, will not be discussed in this paper. Numerical examples are given in section 4 to show the efficiency of the method.

\section{Optimal finite Element SOlution}

Let $V$ be a Hilbert space. Let $a(\cdot, \cdot)$ be a symmetric bilinear functional defined on $V \times V$ satisfying

$$
\alpha\|u\|_{V}^{2} \leq a(u, u) \leq M\|u\|_{V}^{2}
$$

where $M \geq \alpha>0$ are constants, and $\|\cdot\|_{V}$ is the norm of $V$. By $(2.1), \sqrt{a(u, u)}$ defines on $V$ an equivalent norm of $u$, which we call the energy norm as it is in many applications. Let $f(\cdot)$ be a bounded linear functional defined on $V$. Define

$$
F(u)=\frac{1}{2} a(u, u)-f(u), \quad \forall u \in V .
$$

Theorem 2.1. Suppose $u$ is a minimizer of $F(\cdot)$ in $V$, that is

$$
F(u)=\inf _{v \in V} F(v) .
$$

Then, we have

$$
a(v-u, v-u)=2(F(v)-F(u)), \quad \forall v \in V .
$$


Proof. Since $u$ is a minimizer of $F(\cdot)$ in $V$, it satisfies

$$
a(u, v)=f(v), \quad \forall v \in V .
$$

Thus, we have

$$
\begin{aligned}
F(v)-F(u) & =\frac{1}{2} a(v, v)-f(v)-\frac{1}{2} a(u, u)+f(u) \\
& =\frac{1}{2} a(v, v)-a(u, v)-\frac{1}{2} a(u, u)+a(u, u) \\
& =\frac{1}{2} a(v-u, v-u) .
\end{aligned}
$$

This proves the theorem.

Theorem 2.2. Suppose that $F(\cdot ; \Omega)$ is of the form (2.2). Suppose $u$ is a minimizer of $F(\cdot ; \Omega)$ in $\mathbb{U}\left(u_{0} ; \Omega\right)$ (with $p=2$ in $(1.2)$ ), and $\left(\bar{u}_{h}, g_{h}\right)$ is a solution of the mesh transformation method (1.3). Let $u_{h}(x)=\bar{u}\left(g_{h}^{-1}(x)\right)$. Then, $u_{h}$ is the optimal finite element approximation with the least error in energy norm, more precisely

$$
a\left(u_{h}-u, u_{h}-u\right)=\inf _{v_{h} \in \mathbb{U}_{h}\left(u_{0} ; \Omega\right)} a\left(v_{h}-u, v_{h}-u\right)
$$

where

$$
\mathbb{U}_{h}\left(u_{0} ; \Omega\right)=\bigcup_{g \in T_{h}(D)} g\left(\mathbb{U}_{h}\left(u_{0} \circ g ; D\right)\right),
$$

is the set of finite element functions defined on all admissible finite element function spaces defined by (1.5) and (1.6).

Proof. The conclusion of the theorem follows directly from Theorem 2.1 and relation (1.9).

Remark 2.1. We point out here that the inequalities in (2.1) are not used in the proofs of theorems 2.1 and 2.2 .

\section{IMPLEMENTATION OF THE METHOD}

The discrete problem (1.3) can be solved by applying the conjugate gradient method. In general, local minimizers can be found in this way. However, noticing that the set $T_{h}(D)$ is not closed because of the constraint det $\nabla g>0$, a.e. in $D$ (see (1.6)) and there is no guarantee that a minimizing sequence will not go to 
the boundary of $T_{h}(D)$, so we can not prove the existence of solutions for the discrete problem (MTM) (see (1.3)) [4, 5]. In fact, numerical experiments show that without mesh quality control the mesh can sometimes become increasingly irregular in the process of minimization and thus severely deteriorate the convergence behavior of the algorithm. The mesh quality can be brought under control by adding the following term into the energy functional $F(\bar{u}, g ; D)$ (see [8] for details)

$$
F_{q}\left(\bar{u}, g ; \alpha_{0}, \alpha_{1}, \alpha_{2}\right) \equiv \alpha_{0}\left[\alpha_{1} F_{q, c}(\bar{u}, g)+\left(1-\alpha_{1}\right) F_{q, u}(\bar{u}, g)+\alpha_{2} F_{q, r}(g)\right],
$$

where, denoting the monitor matrix by $B \equiv \nabla g(\bar{x})^{T}\left[I+\nabla u \nabla u^{T}\right] \nabla g(\bar{x})$ which is the metric matrix of the deformed body defined on the computational domain,

$$
F_{q, c}(\bar{u}, g) \equiv \int_{D}\left(\frac{1}{n} \operatorname{tr}(B)-(\operatorname{det}(B))^{1 / n}\right) \operatorname{det}(\nabla g) d \bar{x}
$$

is the conformity (or isotropy) control term $[6,7]$,

$$
F_{q, u} \equiv|\Omega|^{1 / 2}\left(\int_{D}(\operatorname{det}(B))^{2 / n} \operatorname{det}(\nabla g) d \bar{x}\right)^{1 / 2}-\int_{D}(\operatorname{det}(B))^{1 / n} \operatorname{det}(\nabla g) d \bar{x}
$$

is the uniformity (or equi-distribution) control term $[6,7]$, and

$$
F_{q, r}(g)=\int_{D}|\log (\operatorname{det} \nabla g)|^{p} d \bar{x}+\int_{D}|\operatorname{det} \nabla g-1|^{s} d \bar{x}
$$

is the regularity control term, where $p, s \in(1, \infty)$ are given constants, and $\alpha_{1} \in[0,1], \alpha_{0}>0, \alpha_{2}>0$ are parameters to control the contributions of the mesh quality control terms.

Define

$$
F_{\alpha}(\bar{u}, g ; D)=F(\bar{u}, g ; D)+F_{q}\left(\bar{u}, g ; \alpha_{0}, \alpha_{1}, \alpha_{2}\right) .
$$

Replacing $F(\bar{u}, g ; D)$ by $F_{\alpha}(\bar{u}, g ; D)$ in $(1.3)$, we obtain the regularized mesh transformation method

$$
(R M T)\left\{\begin{array}{l}
\text { find }\left(\bar{u}_{h}, g_{h}\right) \in \mathbb{U}_{h}\left(u_{0} \circ g_{h} ; D\right) \times T_{h}(D) \text { such that } \\
F_{\alpha}\left(\bar{u}_{h}, g_{h} ; D\right)=\inf _{(\bar{u}, g) \in \mathbb{U}_{h}\left(u_{0} \circ g ; D\right) \times T_{h}(D)} F_{\alpha}(\bar{u}, g ; D) .
\end{array}\right.
$$

Obviously, larger $\alpha_{0}$ implies stronger requirement on the mesh quality, and the mesh transformation method corresponds to $\alpha_{0}=0$.

It is worth noticing that the mesh quality control is used here to guarantee the existence of a solution and to improve the convergence behavior of the minimizing 
procedure. The purpose of mesh transformation is to lower the energy as much as possible by mesh re-distribution, and this is the key issue that differs the mesh transformation method from the moving mesh method (see [6, 7] among many others) where the mesh is redistributed according to the so called equi-distribution principle for the numerical solution obtained. In application, the parameter $\alpha_{0}$ is set initially to a value so that the value of $F_{q}\left(\bar{u}^{0}, g^{0} ; \alpha_{0}, \alpha_{1}, \alpha_{2}\right)$ is comparable to that of $\left|F\left(\bar{u}^{0}, g^{0} ; D\right)\right|$, and is reduced gradually to zero in the minimizing process.

We have the following existence theorem for the regularized mesh transformation method (RMT) (see (3.6)):

Theorem 3.1. Let $\mathfrak{T}_{h}(D)$ be a regular triangulation of D. Suppose the elastic energy density $W(\cdot, \cdot, \cdot)$ is continuous and satisfies the inequality

$$
C_{1}+C_{2}\|\nabla u\|_{p}^{p} \leq \int_{\Omega} W(x, u(x), \nabla u(x)) d x
$$

for all $u \in \mathbb{U}\left(u_{0} ; \Omega\right)$ and for some constants $C_{1} \in R^{1}, C_{2}>0$ and $p>1$. Then, the discrete problem of the regularized mesh transformation method (3.6) has at least one solution for any given parameter $\alpha_{1} \in[0,1], \alpha_{0}>0, \alpha_{2}>0$.

Proof. Noticing that for a given regular triangulation $\mathfrak{T}_{h}(D)$ the element volumes are bounded both from below and above by a positive number, thus by the property of the relative element volume control term $F_{q, r}$, we conclude that $\operatorname{det} \nabla g_{h}$ and $\left(\operatorname{det} \nabla g_{h}\right)^{-1}$ are bounded for a minimizing sequence. On the other hand, by the inequality (3.7), a minimizing sequence of $F_{\alpha}$ in $\mathbb{U}_{h}\left(u_{0} \circ g_{h} ; D\right) \times T_{h}(D)$ is bounded. Thus, the conclusion of the theorem follows from the standard compactness argument and the continuity of $F_{\alpha}$ which is a consequence of $W(\cdot, \cdot, \cdot)$ being continuous.

Theorem 3.2. For fixed $\alpha_{1} \in[0,1]$ and $\alpha_{2}>0$, we have

$$
\lim _{\alpha_{0} \rightarrow 0^{+}} \inf _{(\bar{u}, g) \in \mathbb{U}_{h}\left(u_{0} \circ g ; D\right) \times T_{h}(D)} F_{\alpha}(\bar{u}, g ; D)=\inf _{(\bar{u}, g) \in \mathbb{U}_{h}\left(u_{0} \circ g ; D\right) \times T_{h}(D)} F(\bar{u}, g ; D) .
$$

Proof. For given $\varepsilon>0$, let $\left(\bar{u}_{h}, g_{h}\right) \in \mathbb{U}_{h}\left(u_{0} \circ g_{h} ; D\right) \times T_{h}(D)$ be such that

$$
F\left(\bar{u}_{h}, g_{h} ; D\right)<\inf _{(\bar{u}, g) \in \mathbb{U}_{h}\left(u_{0} \circ g ; D\right) \times T_{h}(D)} F(\bar{u}, g ; D)+\varepsilon .
$$


Since for fixed $\left(\bar{u}_{h}, g_{h}\right), F_{q}\left(\bar{u}_{h}, g_{h} ; \alpha_{0}, \alpha_{1}, \alpha_{2}\right) \rightarrow 0$ as $\alpha_{0} \rightarrow 0$, we have

$$
\lim _{\alpha_{0} \rightarrow 0^{+}} F_{\alpha}\left(\bar{u}_{h}, g_{h} ; D\right) \leq \inf _{(\bar{u}, g) \in \mathbb{U}_{h}\left(u_{0} \circ g ; D\right) \times T_{h}(D)} F(\bar{u}, g ; D)+\varepsilon .
$$

Since $\varepsilon>0$ is arbitrary and $F_{q}$ is non-negative, the inequality (3.10) implies the equation (3.8).

By the definition of $F_{\alpha}$ and the fact that $F_{q}$ is non-negative, Theorem 3.2 implies that the solutions $\left(u_{h}^{\alpha}, g_{h}^{\alpha}\right)$ of (RMT) is a minimizing set and hence is also a bounded set in $\mathbb{U}_{h}\left(u_{0} \circ g ; D\right) \times T_{h}(D)$ as $\alpha$ reduces to zero, that is

$$
\lim _{\alpha \rightarrow 0^{+}} F\left(u_{h}^{\alpha}, g_{h}^{\alpha} ; D\right)=\inf _{(\bar{u}, g) \in \mathbb{U}_{h}\left(u_{0} \circ g ; D\right) \times T_{h}(D)} F(\bar{u}, g ; D) .
$$

Since $\mathbb{U}_{h}\left(u_{0} \circ g ; D\right) \times T_{h}(D)$ is of finite dimension, we have, by extracting subsequences, that $\left(u_{h}^{\alpha}, g_{h}^{\alpha}\right)$ converge to finite element solutions of (MTM). This together with the approximation property of the mesh transformation method $[4,5]$ show the convergence of the regularized mesh transformation method.

\section{Numerical EXAMPLES}

Let $\Omega=D=(-1,1)^{2}$, and let $\mathfrak{T}_{h}(D)$ be a family of regular triangulations of $D$, where $h=h_{N}=2 \sqrt{2} / N$ with $N \geq 2$, introduced by the lines

$$
\begin{cases}x=-1+\frac{2}{N} i, & 0 \leq i \leq N \\ y=-1+\frac{2}{N} j, & 0 \leq j \leq N \\ y= \pm\left(x+2-\frac{4}{N} k\right), & 0<k<N\end{cases}
$$

Let the energy functional $F(u ; \Omega)$ be given by

$$
F(u ; \Omega)=\frac{1}{2} \int_{\Omega}|\nabla u|^{2} d x-\int_{\Omega} f u d x
$$

and the admissible function space $\mathbb{U}\left(u_{0} ; \Omega\right)$ be given by

$$
\mathbb{U}\left(u_{0} ; \Omega\right)=\left\{u \in H^{1}(\Omega): u=u_{0}, \text { on } \partial \Omega\right\} .
$$

Our numerical experiments showed that the numerical results are not very sensitive to the choice of $\alpha_{1}$ and $\alpha_{2}$, however, a better choice can more efficiently bring the mesh quality under control and at the same time leave enough room for the mesh to be transformed. In the following numerical experiments, we set the initial value of $\alpha_{0}$ to $10^{-2}$ and set $\alpha_{1}=0.5, \alpha_{2}=10^{3}$ in the mesh quality 
control term $F_{q}\left(\bar{u}_{h}, g_{h} ; \alpha_{0}, \alpha_{1}, \alpha_{2}\right)$, and we take $p=2$ and $s=4$ in (3.4), and since the function $f$ varies rapidly in $\Omega$, we use a ten point quadrature rule on each element to compute the integral $\int_{\Omega} f u d x$ to limit the inference of the quadrature error. Even though the conjugate gradient method usually leads only to a local optimal mesh distribution, numerical experiments show that the error can often be significantly reduced.

Example 1. Let $k>0$ and $a>0$ be given constants. Let $u_{0}=0$ and

$$
f(x, y)=-\Delta\left[\left(1-x^{2}\right)\left(1-y^{2}\right)\left(w^{2}(\xi)-1\right)\right],
$$

where $\Delta=\partial^{2} / \partial x^{2}+\partial^{2} / \partial y^{2}, \xi=a(k x+y)$ and

$$
w(\xi)=\frac{\exp (\xi)-\exp (-\xi)}{\exp (\xi)+\exp (-\xi)}
$$

Then, $u(x, y)=\left(1-x^{2}\right)\left(1-y^{2}\right)\left(w^{2}(a(k x+y))-1\right)$ is the minimizer of $F(\cdot ; \Omega)$ in $\mathbb{U}\left(u_{0} ; \Omega\right)$.

Figure 1 shows the convergence behavior of finite element solutions, where $e_{r}(F)=e_{r}(F(v ; \Omega))=|F(v ; \Omega)-F(u ; \Omega)| /|F(u ; \Omega)|$ is the relative error on the value of the energy, and where $F(u ; \Omega)$ is obtained by extrapolation on the numerical results assuming that $F\left(u_{h} ; \Omega\right)-F(u ; \Omega) \approx C_{1} h^{2}+C_{2} h^{3}+C_{3} h^{4}$. By Figure 1 and (2.4), we see that $\left|u_{h}^{m}-u\right|_{1,2} \approx O\left(N^{-1}\right)$, that is the energy norm of the error is proportional to the inverse of the square root of the total number of nodes in the mesh.

Fig. 2 shows the final mesh distribution for the case $a=4.0, k=2.0$ and $N=30$, and fig. 3 shows the corresponding finite element solution $u_{h}^{m}$, where we see that the mesh is transformed to allow the finite element functions to have larger variation in certain direction in a particular region which is in agreement with the requirement of the energy minimizer.

Example 2. Let $a>0$ and $k>0$ be given constants. Let

$$
f(x, y)=-\Delta w(\xi)
$$

where $\Delta=\partial^{2} / \partial x^{2}+\partial^{2} / \partial y^{2}, \xi=a\left(x^{2}+y^{2}-k^{2}\right)$ and $w(\xi)$ is defined by (4.4). Let $u_{0}(x, y)=w\left(a\left(x^{2}+y^{2}-k^{2}\right)\right)-1$ for $(x, y) \in \partial \Omega$. Then, $u(x, y)=w\left(a\left(x^{2}+\right.\right.$ $\left.\left.y^{2}-k^{2}\right)\right)-1$ is the minimizer of $F(\cdot ; \Omega)$ in $\mathbb{U}\left(u_{0} ; \Omega\right)$. 
Figure 4 shows the convergence behavior of finite element solutions for Example 2, where we see again that the energy norm of the error is proportional to the inverse of the square root of the total number of nodes in the mesh. We can also see in Figure 4 that, as the parameter $a$ increases, that is as the solutions having sharper variants, the accuracy of the numerical results obtained on the uniform meshes becomes poorer and poorer, while the accuracy of the numerical results obtained by the regularized mesh transformation method is essentially independent of the parameter $a$.

Fig. 5 shows the final mesh distribution for the case $a=12.0$ and $k=0.5$, and fig. 6 shows the corresponding finite element solution $u_{h}^{m}$, where we see again that the mesh is transformed to admit finite element functions which are more flexible in certain directions in some particular region and that leads to a better approximation of the energy minimizer.

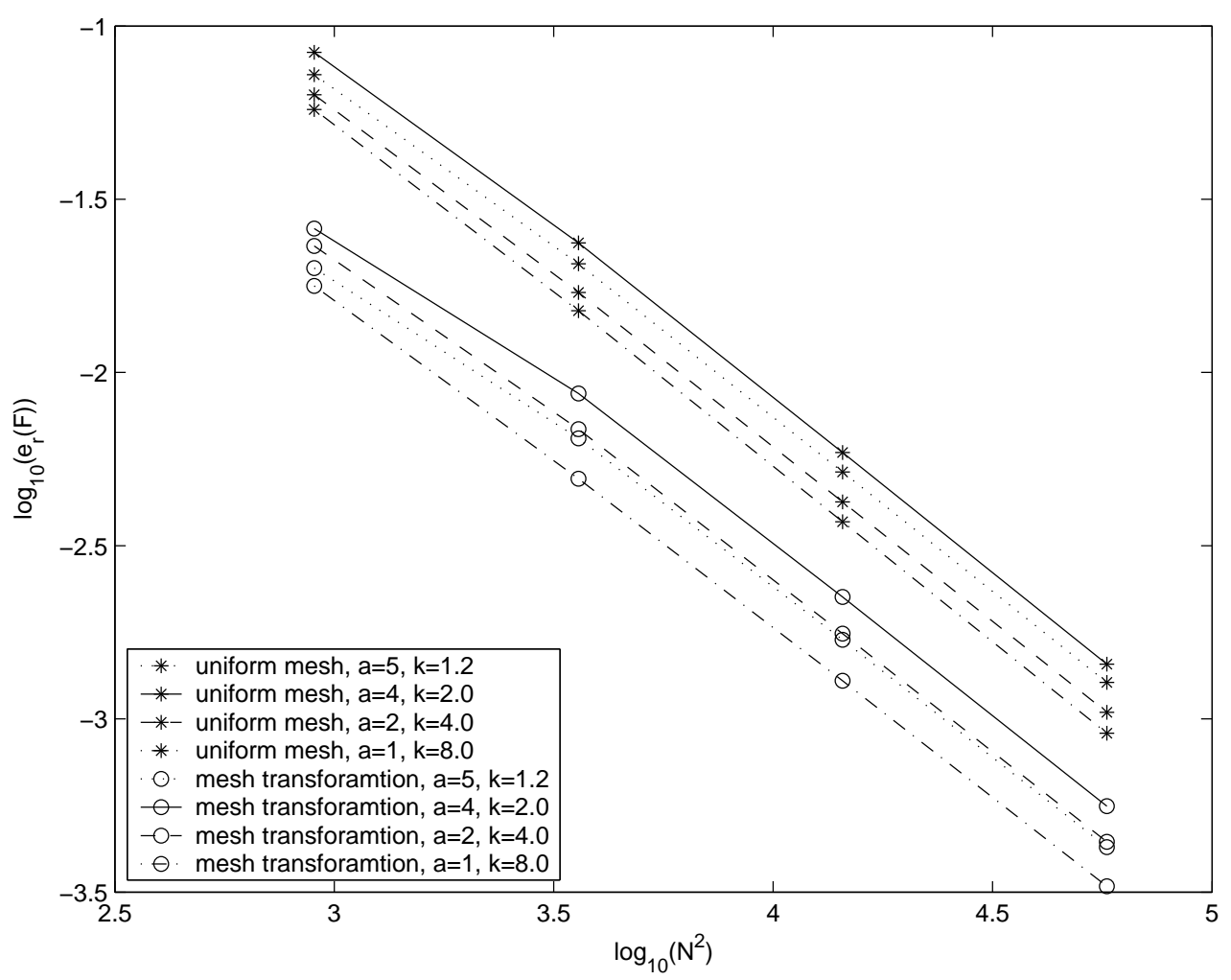

FiguRE 1. The convergence behavior of FEM solutions for Example 1 


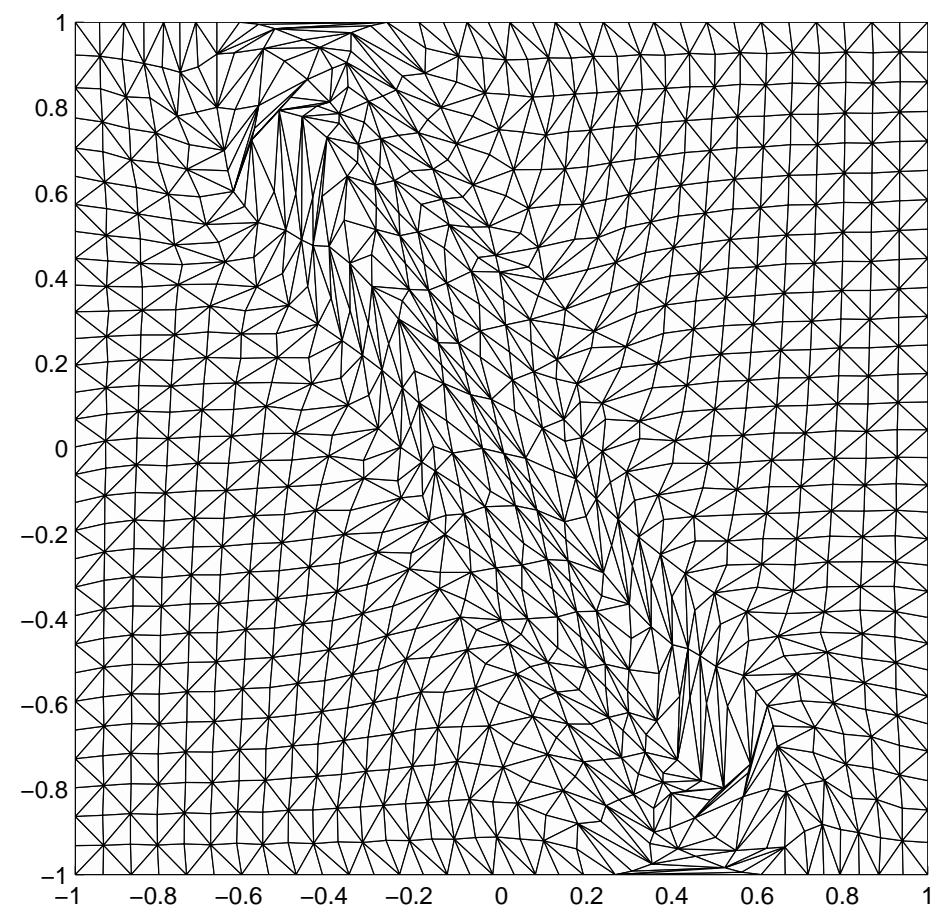

Figure 2. A local optimal mesh for $a=4.0$ and $k=2.0$ for Example 1

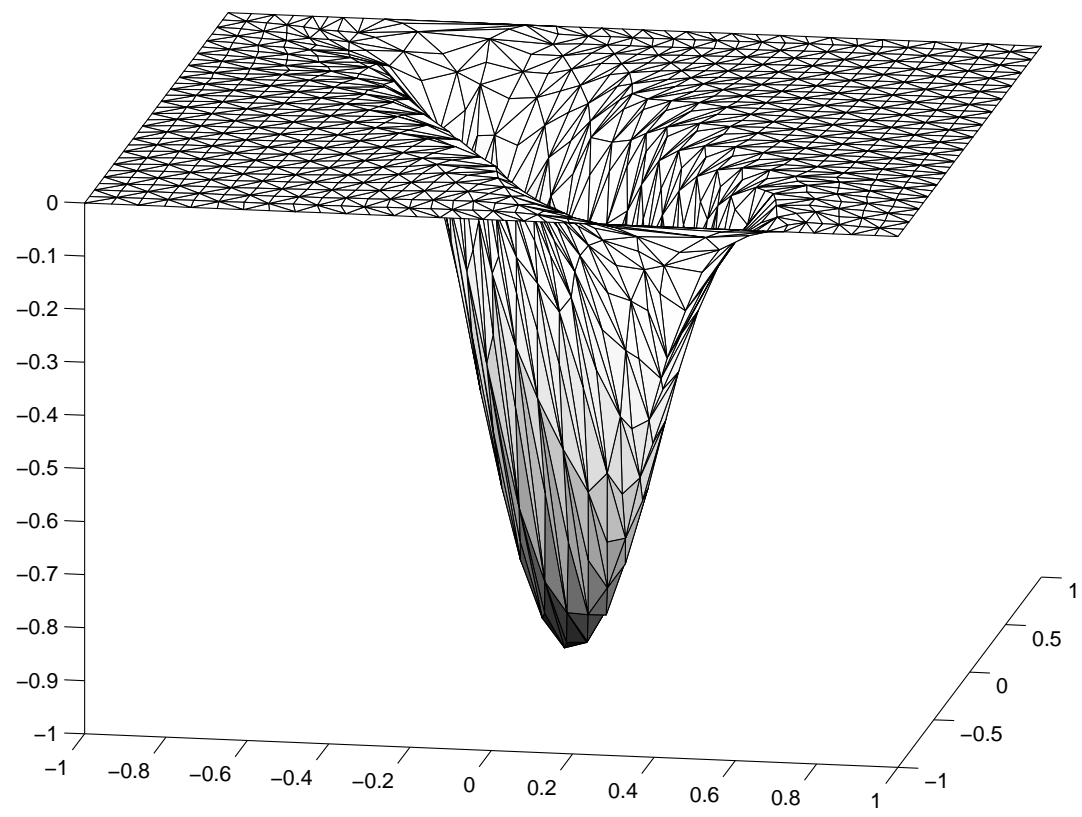

FiguRE 3. $u_{h}^{m}$ for $a=4.0$ and $k=2.0$ for Example 1 


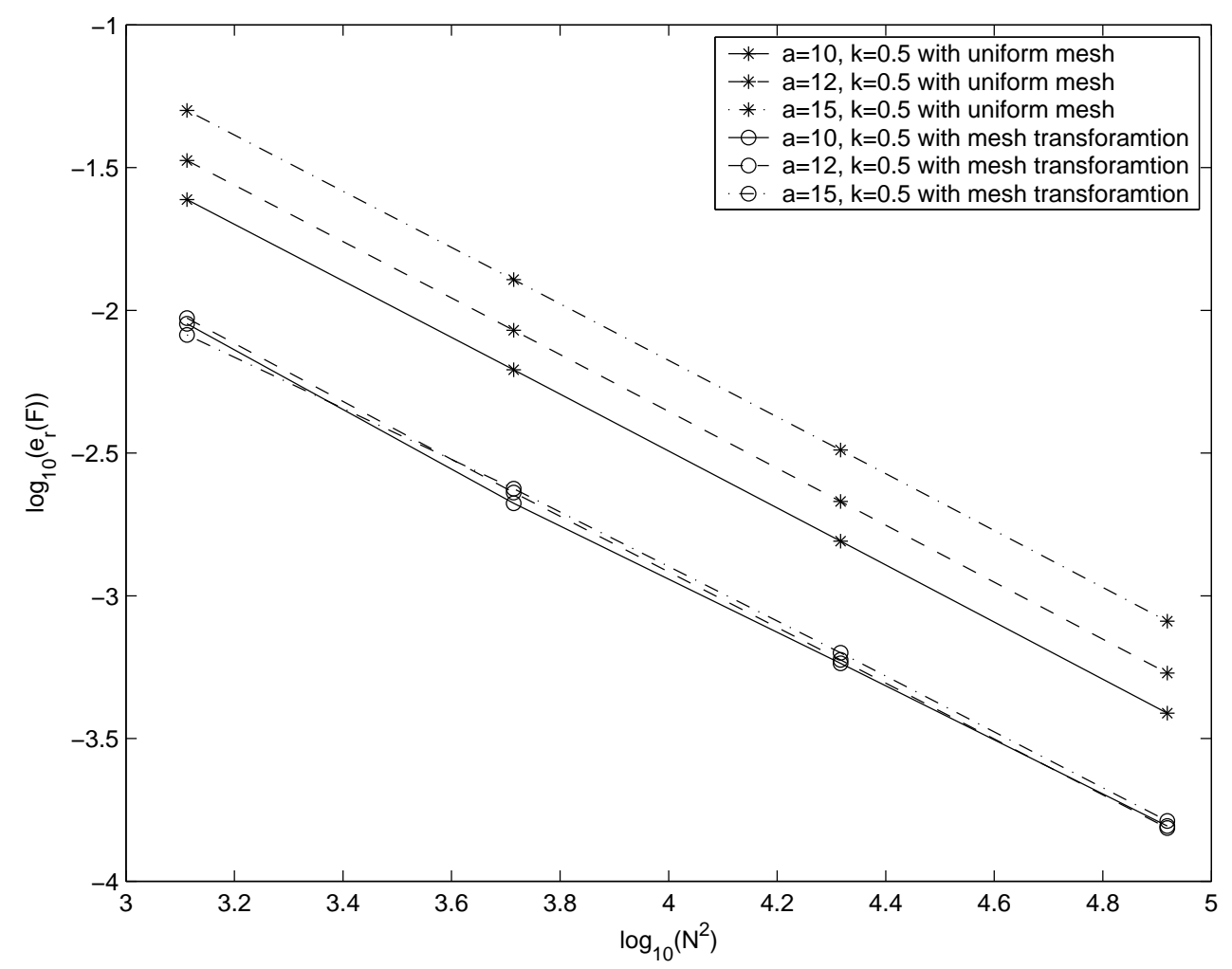

FiguRE 4. The convergence behavior of FEM solutions for Example 2

Remark 4.1. To reduce the error caused by the numerical quadrature, the size of the elements where $f$ and $u$ vary rapidly should not be allowed to become too large, the regularity control term $F_{q, r}(g)$ plays an important role here.

Remark 4.2. How to find the global optimal mesh is an open problem. However, in applications, it is not really necessary to find the global optimal mesh, a local optimal mesh can usually reduce the error efficiently, as is shown in Figure 1 and Figure 4.

Remark 4.3. As we see in the numerical results that the mesh transformation can generate triangles with large angles, however, this will not lead to inconsistent approximation as might happen in general if such triangles are not properly oriented, since the mesh is optimized, at least locally, to reduce the approximation error, which guarantees the approximation error in gradients are small. 


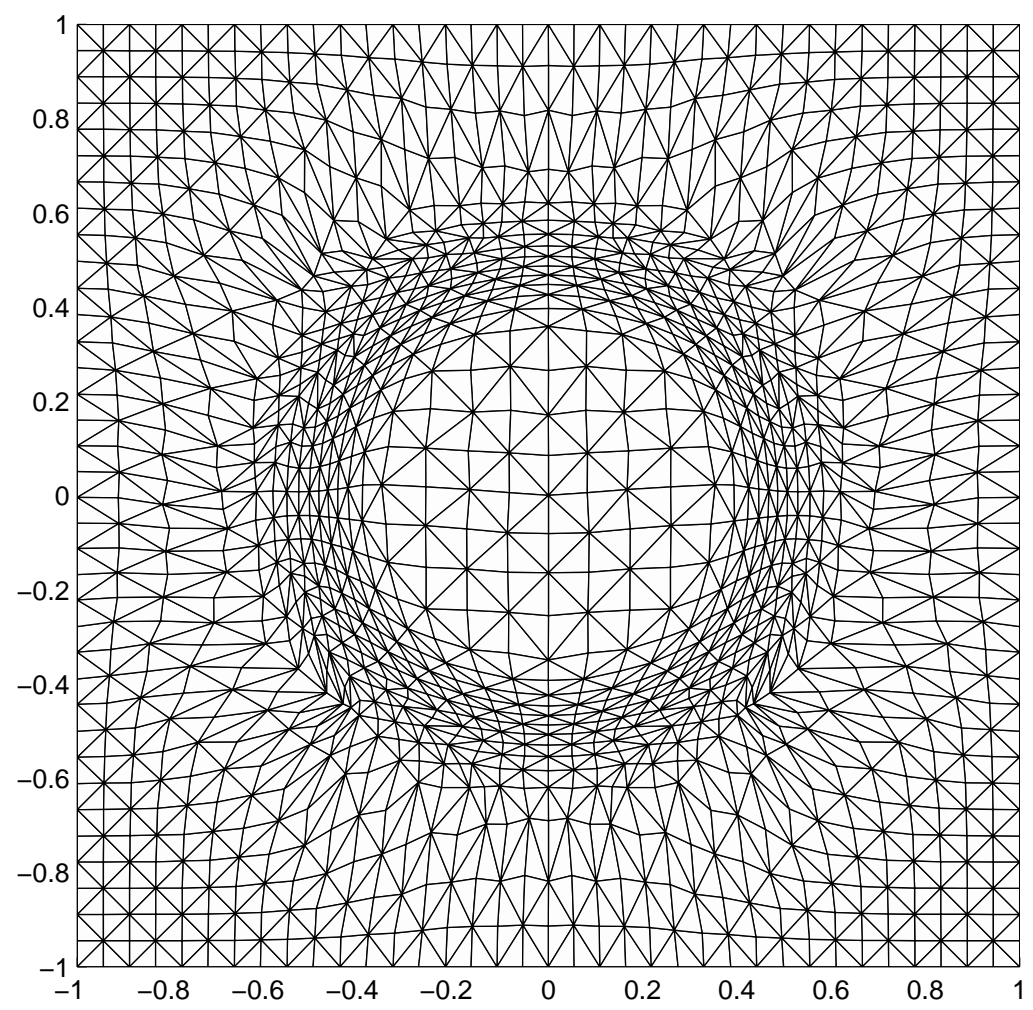

Figure 5. A local optimal mesh for $a=12.0$ and $k=0.5$ for Example 2

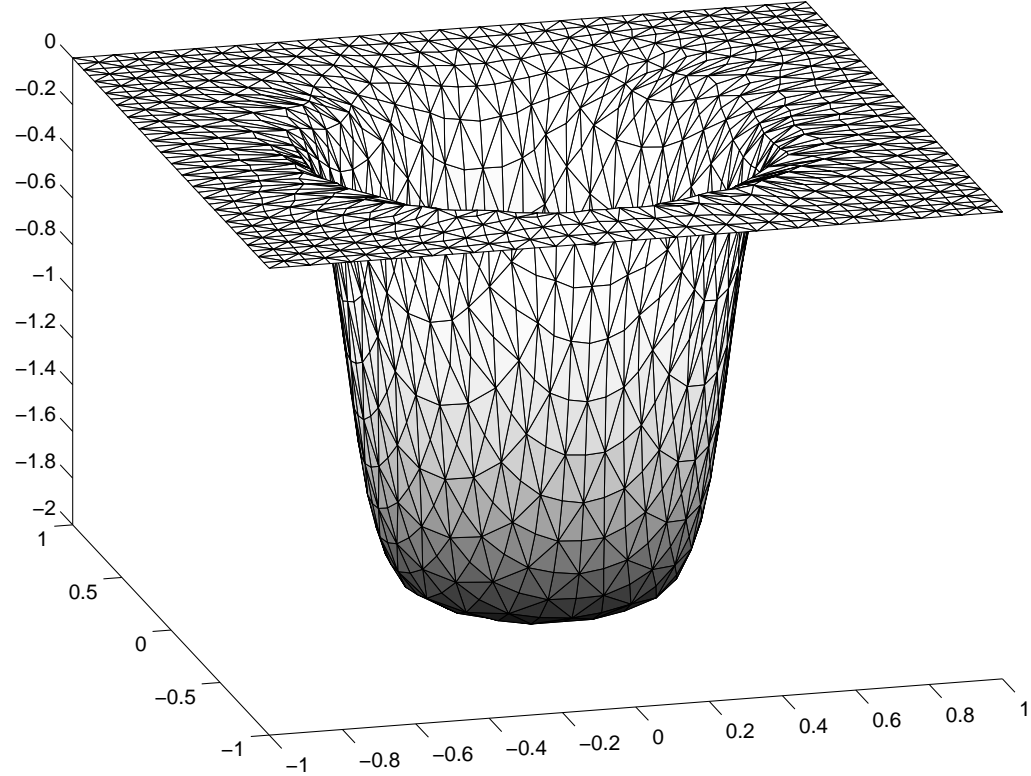

FiguRE $6 . u_{h}^{m}$ for $a=12.0$ and $k=0.5$ for Example 2 


\section{REFERENCES}

[1] B. Dacorogna, Direct Methods in the Calculus of Variations, Applied Mathematical Sciences, 78, Springer-Verlag, Berlin, 1989.

[2] P.G. Ciarlet, The Finite Element Method for Elliptic Problems. North-Holland, Amsterdam, 1978.

[3] Z.-P. Li, Rotational transformation method and some numerical techniques for the computation of microstructures. Math. Models Meth. Appl. Sci., 1998, 8: 985-1002.

[4] Z.-P. Li, A periodic relaxation method for computing microstructures. Appl. Numer. Math., 2000, 32: 291-303.

[5] Z.-P. Li, A mesh transformation method for computing microstructures. Numer. Math., 2001, 89: 511-533.

[6] W. Huang, Practical aspect of formulation and solution of moving mesh partial differential equations. J. Comput. Physics, 2001, 171: 753-775.

[7] W. Huang, Variational mesh adaption: isotropy and equidistribution. J. Comput. Physics, 2001, 174: 903-924.

[8] Z.-P. Li, Regularized mesh transformation method for computation of microstructures. Research report No 51. , Institute of Mathematics and School of Mathematical Sciences, Peking University, 2003.

E-mail address: lizp@math.pku.edu.cn 